This item was submitted to Loughborough's Research Repository by the author.

Items in Figshare are protected by copyright, with all rights reserved, unless otherwise indicated.

\title{
Application of additive manufacturing to the digital restoration of
} archaeological artefacts

PLEASE CITE THE PUBLISHED VERSION

VERSION

AM (Accepted Manuscript)

LICENCE

CC BY-NC-ND 4.0

\section{REPOSITORY RECORD}

Zhang, Fangjin, R.I. Campbell, and lan J. Graham. 2019. "Application of Additive Manufacturing to the Digital Restoration of Archaeological Artefacts". figshare. https://hdl.handle.net/2134/14358. 
This item was submitted to Loughborough's Institutional Repository (https://dspace.lboro.ac.uk/) by the author and is made available under the following Creative Commons Licence conditions.

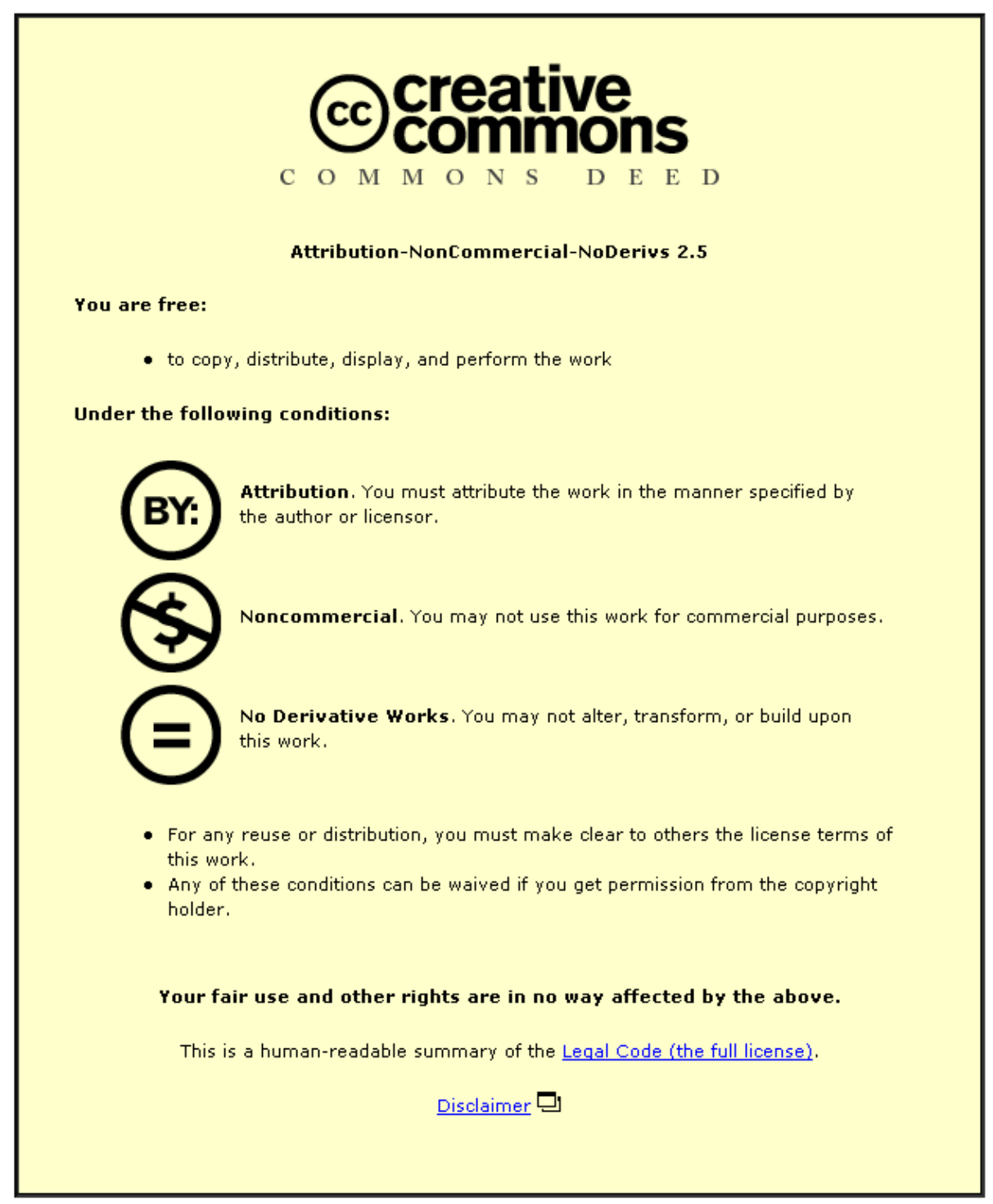

For the full text of this licence, please go to: http://creativecommons.org/licenses/by-nc-nd/2.5/ 


\title{
Application of Additive Manufacturing to the Digital Restoration of Archaeological Artefacts
}

\author{
F. ZHANG ${ }^{1}$, R.I. CAMPBELL ${ }^{2}$ and I.J. GRAHAM ${ }^{3}$
}

1. Loughborough Design School, Loughborough University, Loughborough, Leicestershire, LE113TU, UK; F.Zhang@lboro.ac.uk

2. Loughborough Design School, Loughborough University, Loughborough, Leicestershire, LE11 3TU, UK; R.I.Campbell@lboro.ac.uk

3. Wolfson School of Mechanical and Manufacturing Engineering, Loughborough University, Loughborough, Leicestershire, LE11 3TU, UK; I.J.Graham@lboro.ac.uk

\begin{abstract}
The application of digital technologies to relic conservation is a common research topic in the field of world cultural heritage. Both the inheritance of traditional techniques and the introduction of advanced technologies depend on the users and their awareness and understanding of cultural heritage. Manufacturing processes are the manifestations of culture and art, and there are always new methods appearing in the historical development. Digital technology is now one of these methods, which inherits cultural aspects, improves efficiency and raises quality. Every technology has advantages and limitations. What is important is developing the advantages and avoiding the weaknesses, integrative utilisation, and designing feasible and effective solutions. This paper explains process chains for optimised archiving, restoration, and replication of archaeological artefacts. It shows the exploration of overlapping areas between 3D digital technologies and traditional art, application examples of optimally combined forward and reverse engineering (RE), and developing prospects in the cultural creative industry. The outputs from the research should prove to be valuable to anyone working in the field of digital restoration, particularly when a physical replica is required. This applies in the archaeological domain but also in any field requiring artistic modelling of complex surfaces.
\end{abstract}

Type of paper: Research paper

Keywords: Additive Manufacturing, Archiving, Restoration, Process chains

Introduction of the authors:

Fangjin ZHANG, PhD student, Design School, Loughborough University; Major Interest: Optimising Additive Manufacturing for Preservation of Archaeological Artefacts.

R. I. Campbell, Professor in Design School, Loughborough University; Director of the Research School of Research, PhD supervisor; Major Interest: CAD/CAM and AM research. I. J. Graham, Research Associate in the Wolfson School of Mechanical and Manufacturing Engineering, Loughborough University, PhD co-supervisor; Major Interest: Architectural Engineering and Construction. 


\section{Introduction}

The preservation of cultural heritage is a common research topic throughout the world. Traditional and modern methods, cultural aspects and technology are always developing in a continuous interchange. China, with its five thousand year old civilisation, has a huge amount of work in heritage preservation. Digital technology is being introduced in a new way, which is now a trend in restoration, since it not only inherits culture but also improves efficiency and quality. Our task is to make the best use of the advantages and to avoid the disadvantages, exploring a new way for cultural heritage preservation by integrating and utilising various resources.

\section{Research questions}

In the context of effectively applying digital technologies and optimising additive manufacturing (AM) for heritage preservation, the research questions can be summarised as follows:

- What should be inherited and optimised in relic restoration and repairing?

- How to orientate interdisciplinary methods of digital parameterisation and intuitive modelling?

- What are the advantages of using AM in archaeological restoration?

- To what extent can 3D digital technologies be accepted in the field of archaeological restoration?

- How to optimally combine RE and CAD/CAM systems to obtain desired results?

\section{Case studies}

Loughborough University in the UK has one of the top additive manufacturing research centres in the world. Their research on applications of direct digital manufacturing is at an internationally leading level in this field. The current cooperation with the Forbidden City/Palace Museum aims to design an entire solution for digital restoration including 3D scanning, data manipulation, AM and post processing., The process chains developed will apply to architecture, furniture, relics, decoration, etc. The research project has obtained support from America, Japan and Britain, and a record of the renovation project in film form has been published by the British Museum.

\subsection{Data collection}

The conventional method of archiving is mainly based on photographing, drawing and annotating. The work is extremely delicate and complicated and requires high levels of technical skills and experience, so that the quality depends greatly on labour effort.

The application of 3D scanning enables the recording process to be more accurate and efficient, and makes the document classification richer, with more details. Specifically, every item can be measured and annotated from any angle, and comparison of the damage process following a timeline provides abundant evidence for the need for preservation work. Meanwhile, virtual data storage allows convenient retrieval and saves physical space. More 
importantly, archaeological experts can focus on the research, identification, classification and editing to improve conservation management levels.

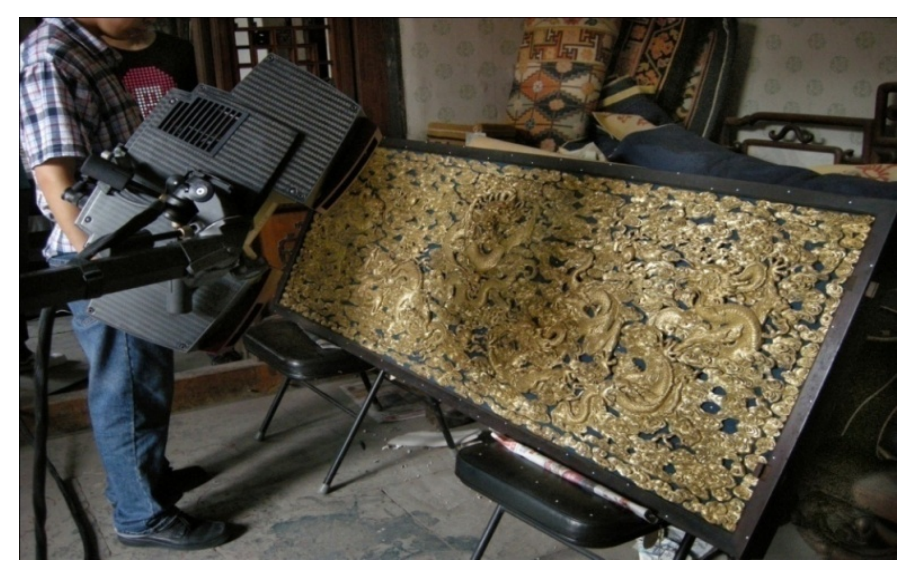

Figure 3.1a 3D scanning for data collocting

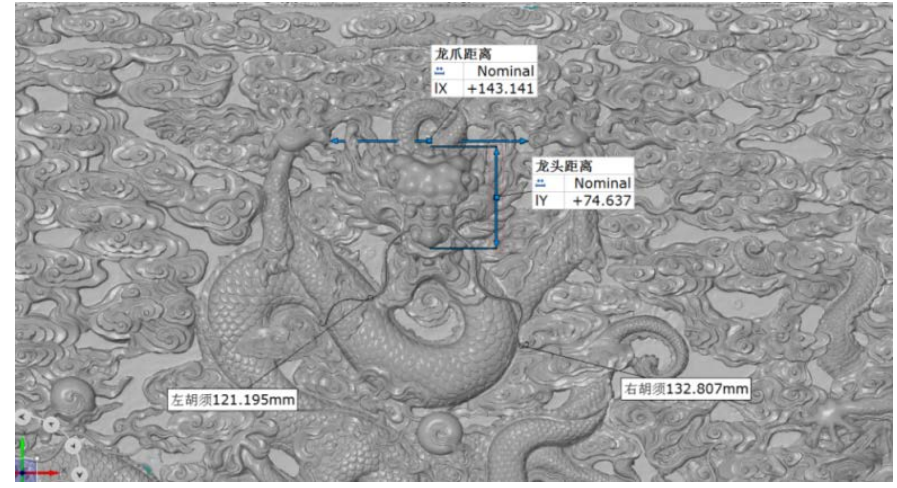

Figure 3.1b Accurate information can be shown on any point of the digital model

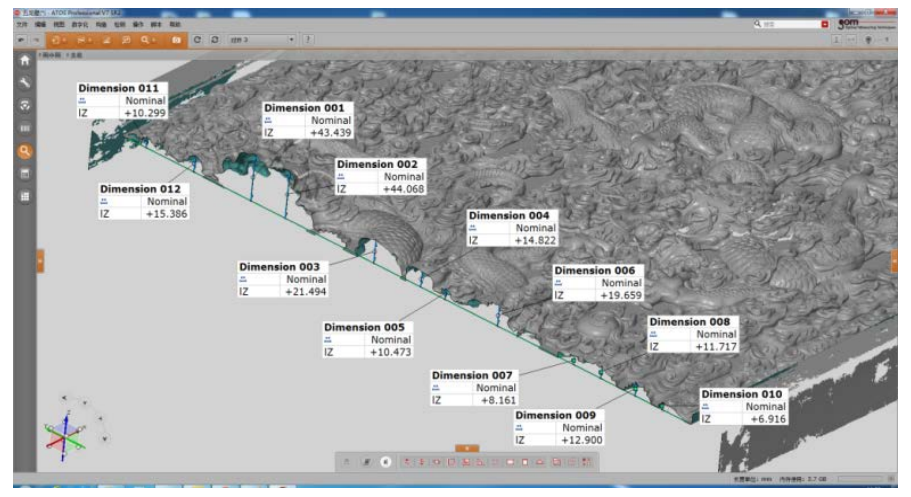

Figure 3.1c Measuring on any sectional view 


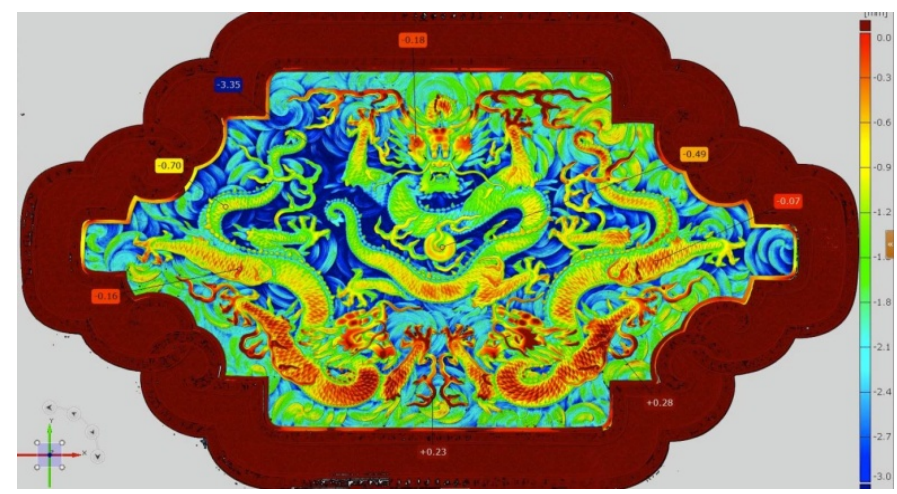

Figure 3.1d Using colour to indicate the deviations on a front view

\subsection{Archiving}

It is possible to manipulate the scanning data with reverse engineering software, such as Geomagic, Freeform and Magics, in order to build 3D digital models and repair them virtually, or to produce required engineering drawing for needs of replication, repair and demonstration.

On one hand, digital archiving ensures accuracy, and precision can be set in different ways according to different needs. For example, large objects, furniture, or even buildings can adopt long distance, large area scanning and be saved as separate sections, which avoids the difficulty of mass data processing and shows the strength of seamless registration. Small scale objects or parts with fine details demand the adoption of high resolution, small area scanning, especially for cracked, corroded and damaged parts, in order to make accurate records and comparison of the changes over time. Also, to obtain true and clear results, different scanners and technologies are applied for different materials and textures, such as highly reflective gold and silver, translucent jade and amber, or unreflective dark fabric surfaces.

On the other hand, the introduction of digital technologies expands the scope of archiving to include standard views and sectional views, inside and outside surfaces, and inner and outer layers analysis. The accurate data can be retrieved flexibly without unnecessary data handling, and demonstrated in 3D animation which shows realistic colours and textures.

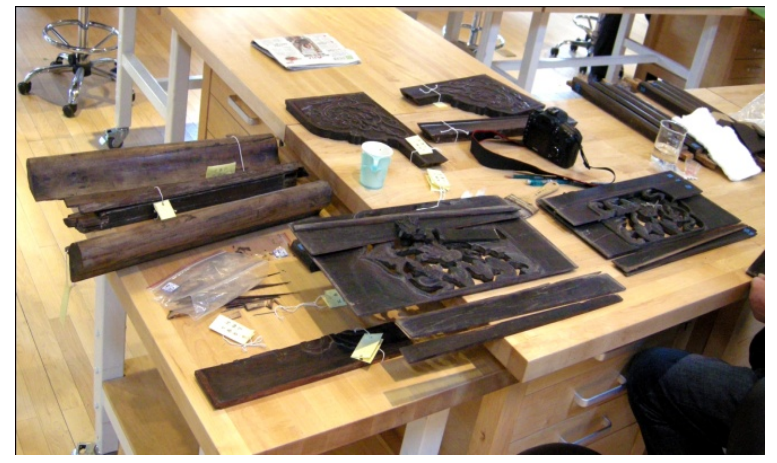

Figure 3.2a Collecting scanning data of furniture parts

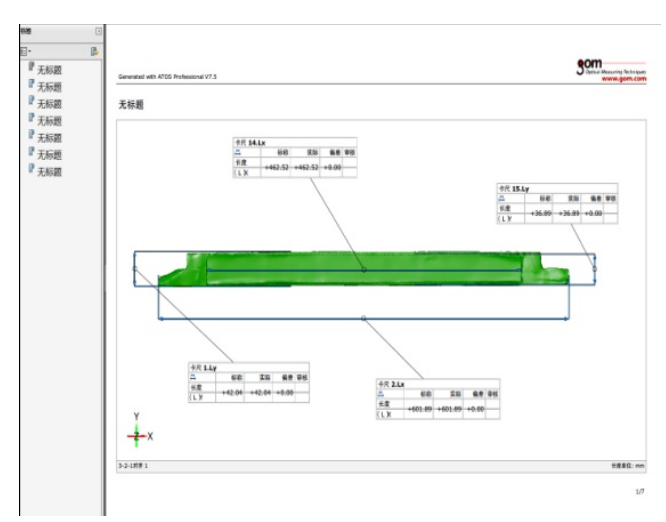

Figure 3.2b 3D digital archive 


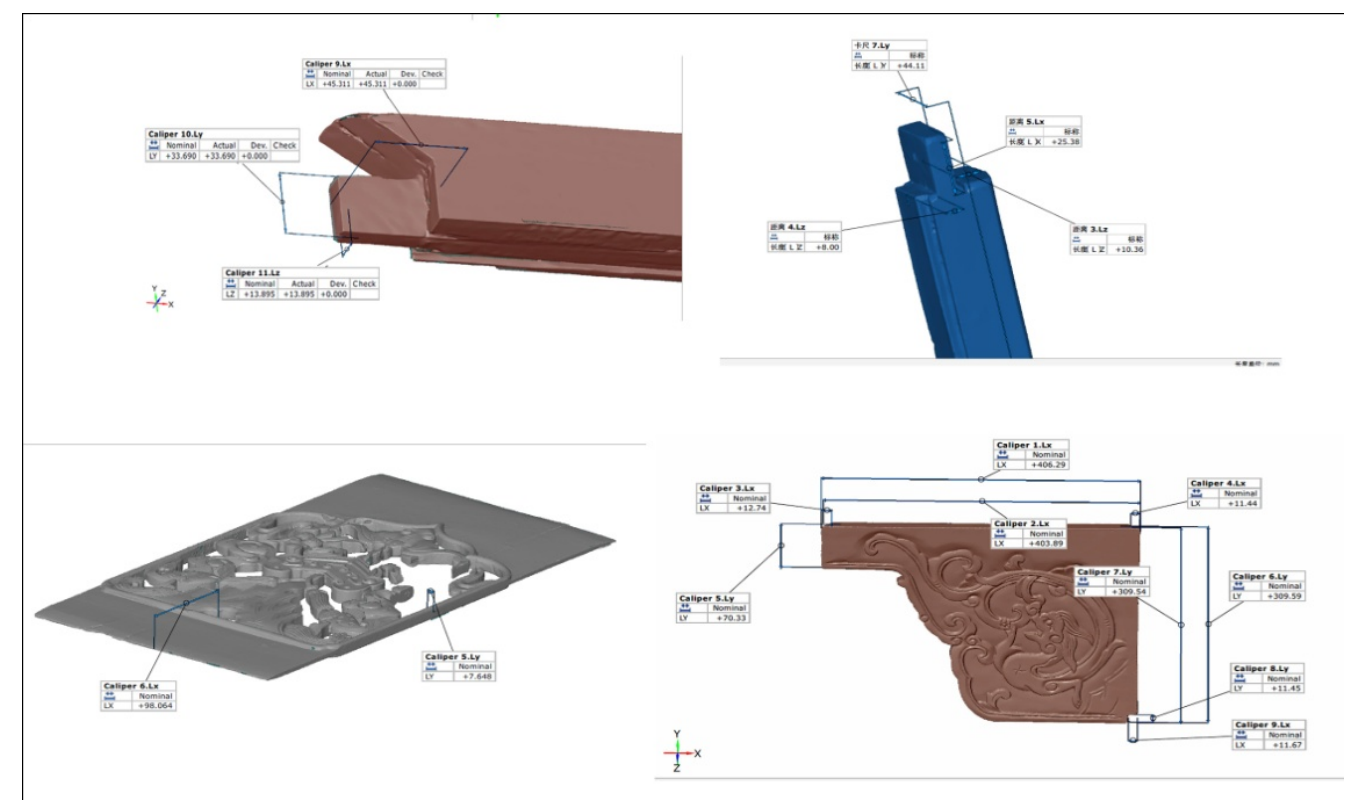

Figure 3.2c 3D coloured model with annotations to guide repair work

\subsection{Restoration}

Every step of relic restoration needs different levels of skilled staff to spend a lot of time working on it, and these skills are normally acquired over many years. The materials can be quite expensive which means any processing mistake may waste a valuable piece of material, increasing time and cost accordingly.

It is easier and more convenient to obtain engineering drawing and digital models by adopting reverse engineering and additive manufacturing, since the requirements for manual skills and time scales are greatly reduced.

3D scanning data enables damaged and missing parts to be previewed virtually in advance, although this needs integration of a lot of knowledge relating to culture and art, e.g. the modelling styles in different historical periods, aging treatments for different materials in different ages. Complex geometry and free-style surfaces require professionals to produce original scaled models for 3D scanning and manipulate the data for digital models. However, additive manufacturing, as a rapid and accurate method, reduces the reliance upon lengthy, repetitive and tedious manual production work. Several kinds of output that meet the requirements have been found. Complex geometry can be produced by additive manufacturing with simulation of surface textures and genuine materials can be produced by CNC machining. Materials for additive manufacturing are increasingly diverse, giving properties such as fine texture, strength, elasticity, colour, transparency, and different compositions in one single build. Also, some genuine materials have been researched and are now available for additive manufacturing, such as copper, stainless steel, wood and ceramics. 

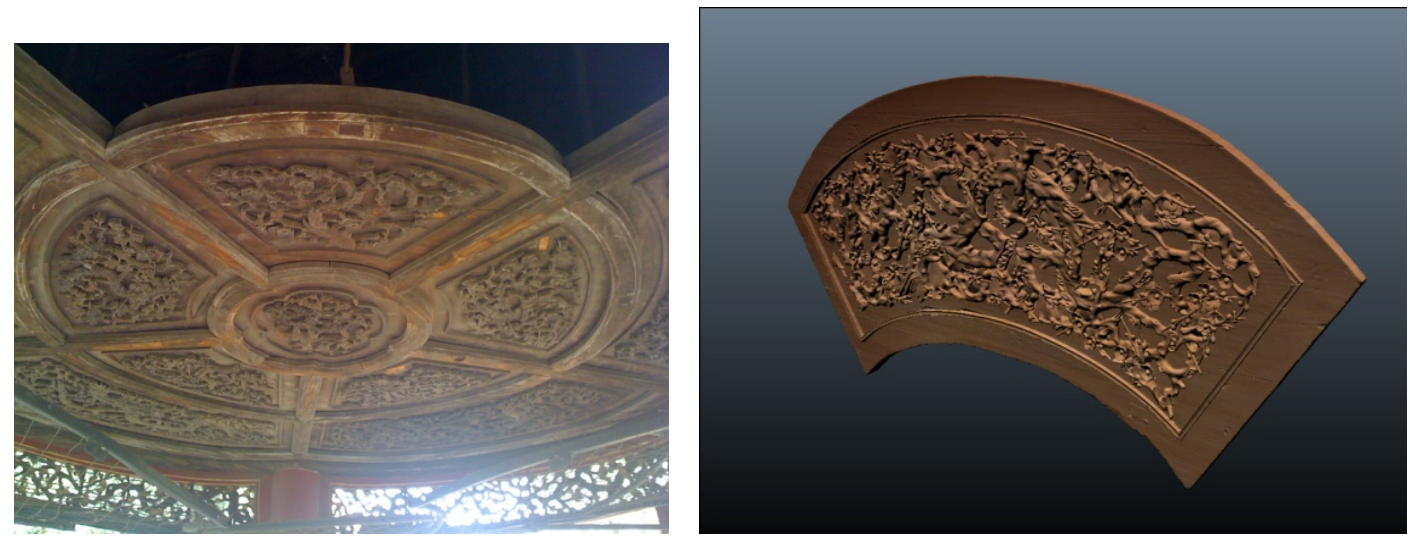

Figure 3.3a A wood ceiling in Chanlong garden Figure 3.3b 3D coloured digital model of ceiling part

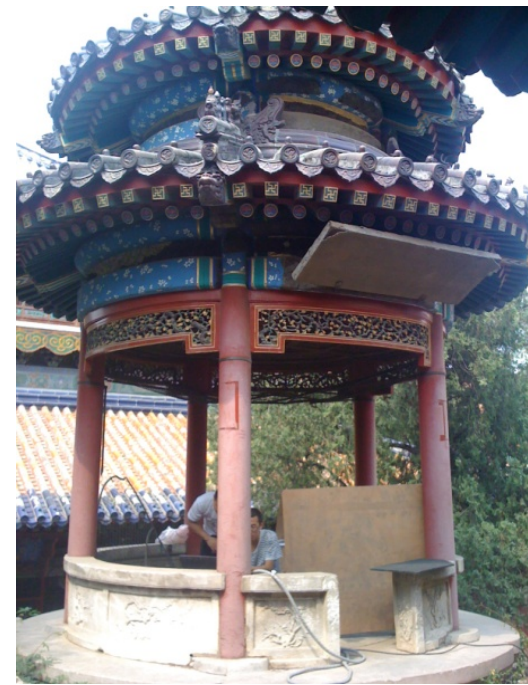

Figure 3.3c The pavilion

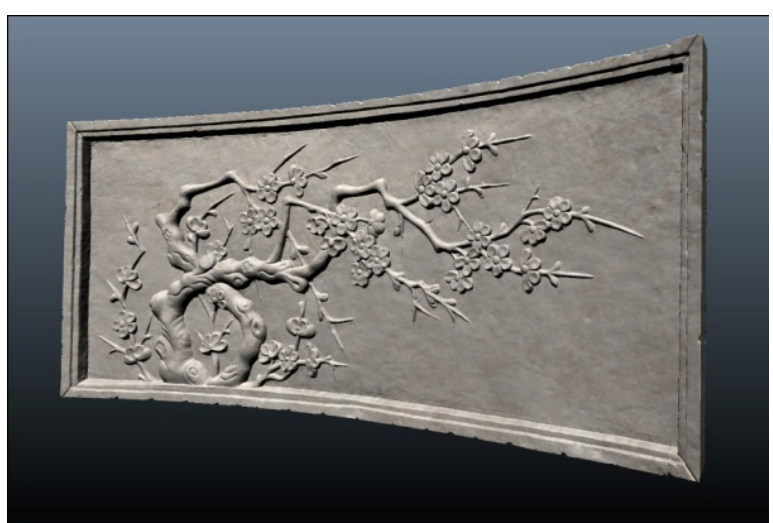

Figure 3.3d 3D coloured digital model of marble enclosure part

\subsection{Downstream product design and manufacturing}

The combination of original data archiving and forward engineering, utilising historical cultural elements and additive manufacturing technologies can produce abundant downstream products. Added value is achieved by the integration of original artefacts' styling features and innovative artistic creation. 


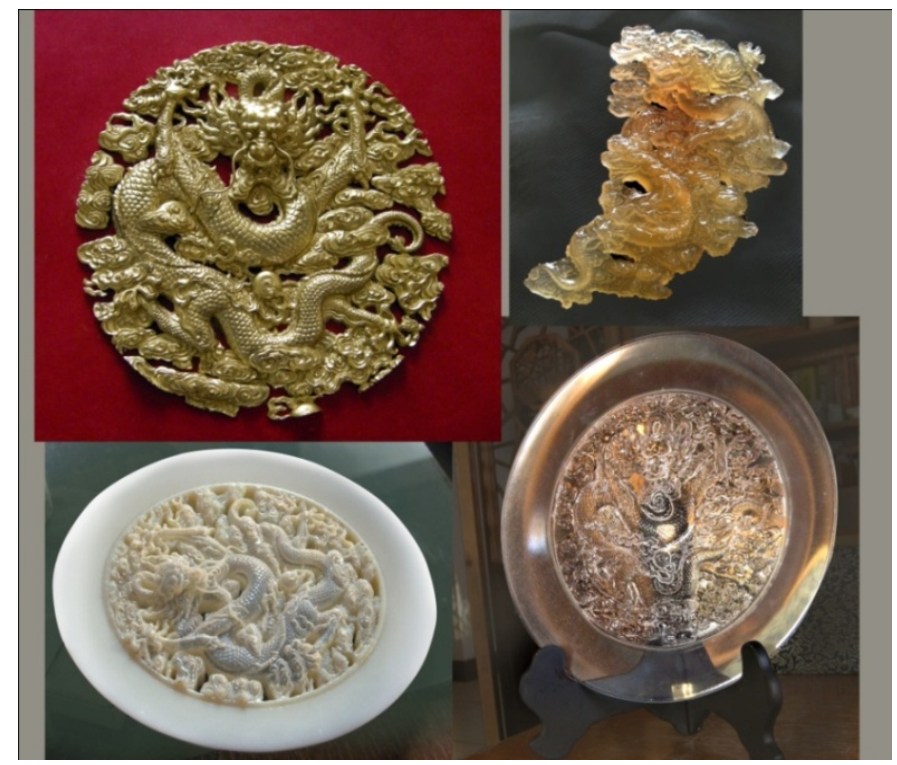

Figure 3.4a Examples of product effects produced by 3D scanning, data manipulation and AM

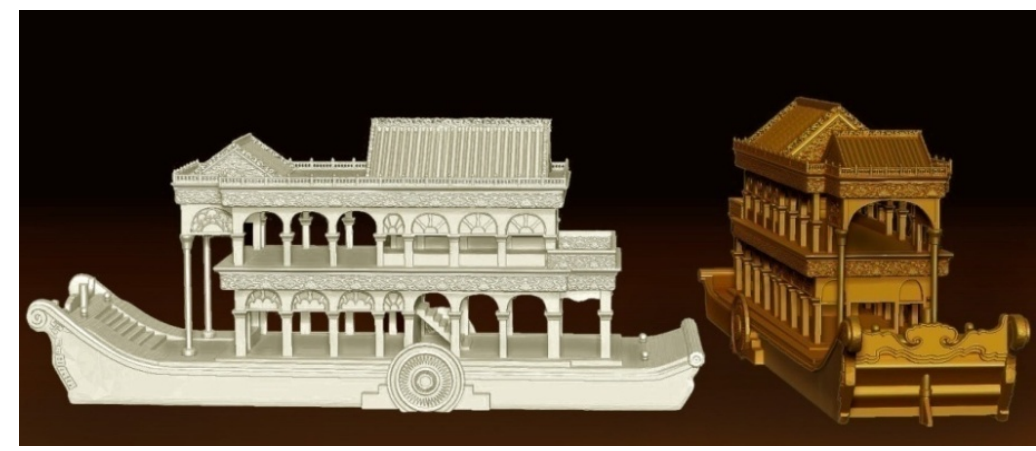

Figure 3.4b Virtual replication of the marble boat in Summer Palace

The original data collected by 3D scanning has more uses, such as demonstration of replicas, images and animation for document publication and films via data manipulation. This involves extracting, managing and creating key cultural elements, development of customised products and decorations with both aesthetics and functionality. In short, digital technologies can play a significant role in the cultural creative industry.

\section{Conclusion}

Digital technologies are gradually being proved by case studies as useful tools for optimising conventional methods, in order to improve the technical levels of cultural heritage preservation. The impact of reverse engineering and additive manufacturing on the cultural art and archaeological preservation fields is similar to the impact of photography on painting in the past two centuries. Digital technologies will not replace skilled work, but will greatly enrich and develop these subjects. However, there are still problems to be solved at this current stage:

o Digital technologies when compared with manual skilled work would somehow change the nature of cultural art

o Communication and coordination between engineering and art subjects

o How to position interdisciplinary subjects and obtain governmental support? 


\section{Future Prospects}

At present, China is still in a critical period of development. Developing advanced technologies that are appropriate to the Chinese condition is our priority. Hence, we keep up with world trends in high-tech development and place it on a strategic priority, striving for an invincible position in the face of fierce market competition. Research on digital technologies is imperative as it comes with promising prospects.

\section{References}

[1] Balc, N., Campbell, R.I., 'From CAD and RP to Innovative Manufacturing', Computing and Solutions in Manufacturing Engineering, Ivan, N.V., Lancea, C., Filip, A., Chicos, L., Mihali, M. and Simon, A.E. (eds), Transylvania University of Brasov, Computing and Solutions in Manufacturing Engineering Conference 2004, Brasov/Sinaia, Romania, 2004, [CD-ROM].

[2] Ferschin, P., Jonas, A., Kulits, I. and Raue, D. (2011) Rapid Prototyping of Digital Models in Archaeology, Conference on Culture Heritage and Technologies, Vienna, November 14-16, 2011.

[3] Louis, C., Lawrence, M. and Keith, M. Research Methods in Education, $6^{\text {th }}$ Edition, Routledge Publications, London, UK, 2007

[4] Weber, J.A., Malone, E., Exporting Virtual Material Culture: Cheap and Easy Methods to Preserve and Share Data, The SAA Archaeological Record, vol. 11, No. 1, pp. 15-18, Society of American Archaeology, Jan. 2011

[5] Williams, M., Laser Scanning in PTM format, Warwick Manufacturing Group, University of Southampton.

http://www.soton.ac.uk/archaeology/acrg/acrg_research_herculaneum_scanning.html

[6] Zhang, F., Campbell, R.I. and Graham, I.J., (2011) Application of additive manufacturing to fine art sculpture, in Proceedings of the 12th Conference on Rapid Design, Prototyping and Manufacturing, Renie, A.E.W., Bocking, C.E. (Eds), CRDM Limited, 12th Conference on Rapid Design, Prototyping and Manufacturing, Lancaster University, 2011, p69-77, ISBN 978-0-9566643-1-0.

\section{Acknowledgement}

Grateful appreciation is given to the following organisations who made this work possible: The Palace Museum, The Summer Palace, China Association for Preservation Technology of Cultural Relics, Central Academy of Fine Arts, Tsinghua University, Beijing University, Road Ahead Technologies Consultant Corporation, I AM Reverse Engineering Company. 